\title{
TU/e emonownen

\section{Dynamics of magnetic chains in a shear flow under the influence of a uniform magnetic field}

\section{Citation for published version (APA):}

Kang, T. G., Hulsen, M. A., \& Toonder, den, J. M. J. (2012). Dynamics of magnetic chains in a shear flow under the influence of a uniform magnetic field. Physics of Fluids, 24(4), 1-19. [042001].

https://doi.org/10.1063/1.4704822

DOI:

$10.1063 / 1.4704822$

Document status and date:

Published: 01/01/2012

\section{Document Version:}

Publisher's PDF, also known as Version of Record (includes final page, issue and volume numbers)

\section{Please check the document version of this publication:}

- A submitted manuscript is the version of the article upon submission and before peer-review. There can be important differences between the submitted version and the official published version of record. People interested in the research are advised to contact the author for the final version of the publication, or visit the $\mathrm{DOI}$ to the publisher's website.

- The final author version and the galley proof are versions of the publication after peer review.

- The final published version features the final layout of the paper including the volume, issue and page numbers.

Link to publication

\section{General rights}

Copyright and moral rights for the publications made accessible in the public portal are retained by the authors and/or other copyright owners and it is a condition of accessing publications that users recognise and abide by the legal requirements associated with these rights.

- Users may download and print one copy of any publication from the public portal for the purpose of private study or research.

- You may not further distribute the material or use it for any profit-making activity or commercial gain

- You may freely distribute the URL identifying the publication in the public portal.

If the publication is distributed under the terms of Article $25 \mathrm{fa}$ of the Dutch Copyright Act, indicated by the "Taverne" license above, please follow below link for the End User Agreement:

www.tue.nl/taverne

Take down policy

If you believe that this document breaches copyright please contact us at:

openaccess@tue.nl

providing details and we will investigate your claim. 


\section{Dynamics of magnetic chains in a shear flow under the influence of a uniform magnetic field}

Tae Gon Kang, Martien A. Hulsen, and Jaap M. J. den Toonder

Citation: Phys. Fluids 24, 042001 (2012); doi: 10.1063/1.4704822

View online: http://dx.doi.org/10.1063/1.4704822

View Table of Contents: http://pof.aip.org/resource/1/PHFLE6/v24/i4

Published by the American Institute of Physics.

\section{Related Articles}

A universal noncontact flowmeter for liquids

Appl. Phys. Lett. 100, 194103 (2012)

Travelling waves in a cylindrical magnetohydrodynamically forced flow

Phys. Fluids 24, 044101 (2012)

Two-dimensional numerical analysis of electroconvection in a dielectric liquid subjected to strong unipolar injection

Phys. Fluids 24, 037102 (2012)

Properties of bubbled gases transportation in a bromothymol blue aqueous solution under gradient magnetic fields

J. Appl. Phys. 111, 07B326 (2012)

Magnetohydrodynamic flow of a binary electrolyte in a concentric annulus

Phys. Fluids 24, 037101 (2012)

\section{Additional information on Phys. Fluids}

Journal Homepage: http://pof.aip.org/

Journal Information: http://pof.aip.org/about/about_the_journal

Top downloads: http://pof.aip.org/features/most_downloaded

Information for Authors: http://pof.aip.org/authors

\section{ADVERTISEMENT}

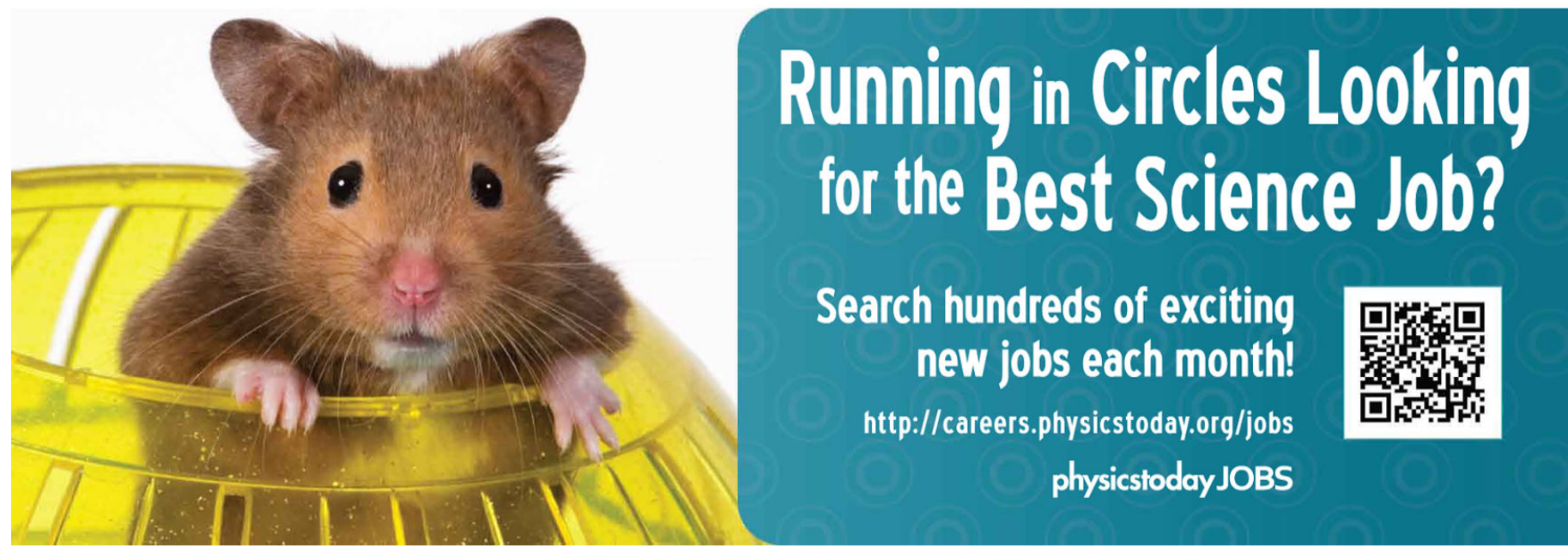




\title{
Dynamics of magnetic chains in a shear flow under the influence of a uniform magnetic field
}

\author{
Tae Gon Kang, ${ }^{1, a)}$ Martien A. Hulsen, ${ }^{2}$ and Jaap M. J. den Toonder ${ }^{2}$ \\ ${ }^{1}$ School of Aerospace and Mechanical Engineering, Korea Aerospace University, \\ 100 Hanggongdae-gil, Hwajeon-dong, Goyang-city, Gyeonggi-do 412-792, South Korea \\ ${ }^{2}$ Department of Mechanical Engineering, Eindhoven University of Technology, P.O. Box 513, \\ 5600 MB Eindhoven, The Netherlands
}

(Received 22 September 2011; accepted 23 March 2012; published online 23 April 2012)

\begin{abstract}
We numerically investigate dynamics of magnetic chains and flow characteristics in a two-dimensional shear flow under the influence of a magnetic field applied externally. A direct simulation method is employed to solve the particulate flow in the creeping flow regime, taking into account both magnetic and hydrodynamic interactions in a coupled manner. In a periodic channel, the dynamics of chains is found to be significantly influenced by the Mason number (the ratio of viscous force to magnetic force), the magnetic susceptibility, and the particle fraction. Below a critical Mason number, a chain rotates and reaches an equilibrium. Above the critical value, however, the chain continuously rotates as a rigid body. Thinning behavior in the wall shear stress is found above a threshold value of the Mason number. As for chain rupture in the shear flow, three regimes of the Mason number are found, showing three typical conformations of the chains: (i) complex chains with branches rather than linear chains, (ii) tilted linear chains broken in the middle, generating a slip zone between the upper and lower chains, and (iii) shortened chains rotating in the channel. (c) 2012 American Institute of Physics. [http://dx.doi.org/10.1063/1.4704822]
\end{abstract}

\section{INTRODUCTION}

Suspensions of magnetic particles are used in a variety of applications. Magnetorheological (MR) fluids, consisting of tiny magnetizable particles suspended in a non-magnetic medium, are widely used in many industrial applications such as brakes, dampers, actuators, etc. ${ }^{1,2}$ Rapid response to the magnetic field applied externally makes MR fluids an attractive means for the devices. Recently, magnetic particles are also employed in microfluidic systems for chemical or biological applications by which our research is motivated. In such applications, magnetic particles are adopted as mobile substrates for bio-assays, stirring agents to achieve efficient mixing, or actuators. ${ }^{3-10}$ Under typical operating conditions, due to small length scale, flows in these devices are laminar and inertia of both fluid and particles may be neglected.

Magnetic particles dispersed in a liquid form chains or clusters upon applying an external magnetic field, generating field-dependent magnetorheological structures due to the external field and magnetic interactions among the particles. When both continuous shearing and a magnetic field are applied, the structures of chain, column, or cluster, consisting of polarizable particles such as paramagnetic particles, are affected by the combined effect of viscous shear forces and magnetic forces, leading to the topological changes such as breakup or reformation of chains. ${ }^{11-15}$ It is well known that, in such a shear flow, rotational dynamics is governed by a dimensionless number called the Mason number, the ratio of viscous force to magnetic force. ${ }^{11,13,16} \mathrm{In}$ a similar way, rotational dynamics of chains driven by a rotating magnetic field is also characterized by the Mason number. ${ }^{8,17,18}$ The motion of an individual particle, formation and dynamics of the such

a) Author to whom correspondence should be addressed. Electronic mail: tgkang@kau.ac.kr. 
magneto-rheological structures, and subsequent fluid flow are key issues from a fundamental point of view, requiring further investigations.

In many previous studies, the particle dynamics method based on the point-dipole approximation and the Stokes drag law has been extensively employed due to its simplicity. ${ }^{9,11,15,17}$ However, this method may lead to quantitatively inaccurate results in the cases with closely packed particles like clusters or chains. More sophisticated numerical schemes based on the Stokesian dynamics are capable of treating multi-body hydrodynamic interactions, but still with an approximation on the magnetic forces. ${ }^{13,14}$ Even though previous methods have been successfully applied to various applications, more complete representations of the field-induced coupling and hydrodynamic interactions among particles are required to understand detailed flow characteristics and chain conformations, especially in microfluidic applications with the effect of geometric confinement. The direct simulation method, ${ }^{19}$ which we recently developed, is the product of an effort to overcome the limitations of previous numerical methods, enabling complete representations of magnetic and hydrodynamic interactions among particles forming complex magneto-rheological structures. In our method, the incorporation of complicated boundary conditions, as in microfluidic channels, is relatively easy compared to the Stokesian dynamics approach. More recently, a direct simulation scheme based on an immersed-boundary finite-volume method was also introduced to solve flows with paramagnetic particles. ${ }^{20}$

We choose a two-dimensional shear flow with magnetic chains suspended in a periodic rectangular channel under the influence of a magnetic field. The magnetic field applied externally is uniform in strength and perpendicular to the shear flow imposed. Magnetic chains are formed by circular paramagnetic particles with a constant magnetic susceptibility and the liquid is a non-magnetic Newtonian fluid. Our main concerns are the rotational dynamics of chains, flow characteristics due to the presence of particles, and chain rupture in the shear flow. Especially, we focus on the effect of the Mason number, the magnetic susceptibility, and the fraction of magnetic particles suspended in the channel.

The direct numerical simulation method, ${ }^{19}$ based on a fictitious domain method and the finite element method, is employed to solve the magnetic particulate flow. The numerical scheme is capable of taking into account both hydrodynamic and magnetic interactions in a coupled manner. The forces resulting from magnetic interactions between particles are implemented via the Maxwell stress tensor ${ }^{21,22}$ and the hydrodynamic interactions are treated by the fictitious domain method. ${ }^{23}$ Therefore, our numerical scheme is thought to be a proper choice to solve dynamics of chains involving mutual magnetic interactions between particles spaced closely and flow characteristics affected by the particle motion and the externally imposed flow.

The paper is organized as follows. First, we introduce the problem and the governing equations to solve the problem. Then, the finite element formulations for the magnetic and flow problems are addressed briefly. A single-chain problem is solved first, focusing on the rotational dynamics characterized by the Mason number and the magnetic susceptibility. Next, the flow characteristics influenced by particle fraction are investigated, with an emphasis on the magneto-viscous effect affected by the Mason number and the susceptibility. Finally, we show chain rupture in the shear flow. Topological changes of the magnetic chains in the shear flow are investigated using the direct simulation method, revealing three regimes of the Mason number showing specific structures of chains depending on the relative importance between the magnetic and the hydrodynamic forces.

\section{MODELING}

\section{A. Problem definition}

The problem we wish to solve is, as schematically illustrated in Fig. 1, the motion of circular magnetic particles and fluid flow in a two-dimensional shear flow under the influence of an externally applied magnetic field. The particles are paramagnetic, i.e., magnetically neutral in the absence of a magnetic field, but magnetized under the influence of an external magnetic field. We neglect the effect of magnetic saturation, which is an approximation that is valid for relatively small field strengths, leading to a linear relation between the applied field strength and the induced magnetic 


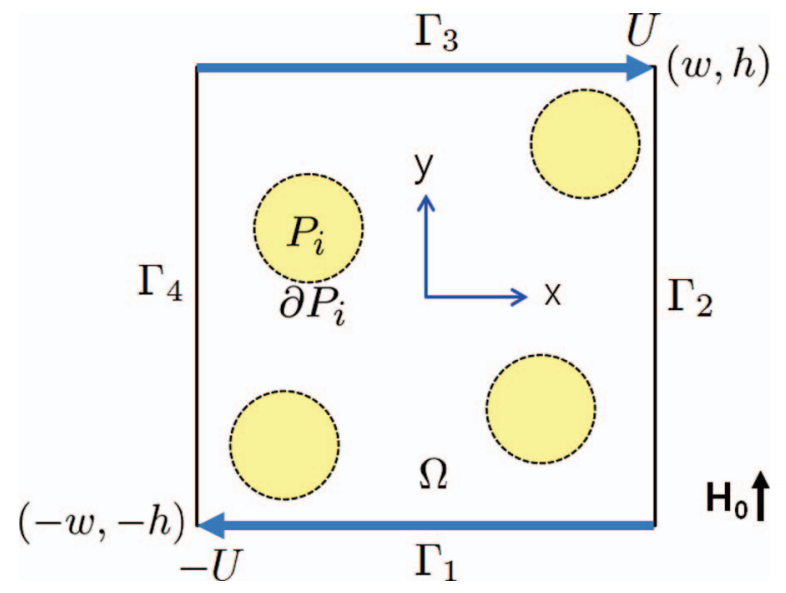

FIG. 1. Magnetic particles suspended in a shear flow under the influence of an externally applied magnetic field $\boldsymbol{H}_{0}$ directed vertically. The circular particles with radius $a$ are paramagnetic with a constant permeability $\mu_{\mathrm{p}}$ and the surrounding medium is a Newtonian fluid with viscosity $\eta$ and permeability $\mu_{\mathrm{f}}$. The origin of the coordinate system is located at the center of the channel, which is periodic in the $x$ direction. The channel is $2 w$ wide and $2 h$ high, and the four boundaries of the channel are represented by $\Gamma_{1}, \Gamma_{2}, \Gamma_{3}$, and $\Gamma_{4}$. A particle is represented by $P_{i}$, the boundary of the particle by $\partial P_{i}$, and the fluid domain by $\Omega$. $U$ and $-U$ are horizontal velocity components at $\Gamma_{3}$ and $\Gamma_{1}$, respectively.

moment. The particles are assumed to be non-Brownian and inertialess, but they interact with the surrounding fluid and other particles both hydrodynamically and magnetically. The fluid is assumed to be non-magnetic and Newtonian with viscosity $\eta$. The constant magnetic permeabilities of the particles and the fluid are $\mu_{\mathrm{p}}$ and $\mu_{\mathrm{f}}$, respectively, where $\mu_{\mathrm{p}}>\mu_{\mathrm{f}}$. Assuming that the magnetic field is generated by static current (or very slowly changing current), we solve magnetostatics, which is a special case of the Maxwell equations in the absence of an electric field and electrostatic charges. ${ }^{24,25}$ Since we are concerned with a flow in microscale, the fluid flow is governed by the viscous force and the magnetic actuation acting on the particles, neglecting the effect of inertia and gravity.

A typical computational domain consists of circular particles all with the same radius $a$ suspended in a rectangular channel filled with a non-magnetic Newtonian fluid. The channel is periodic in the horizontal direction, i.e., a particle that exits through a periodic boundary (either $\Gamma_{2}$ or $\Gamma_{4}$ in Fig. 1) can reenter the domain through the other boundary. Particles are denoted by $P_{i}(t)(i=1, \ldots, N)$, where $N$ is the number of particles in the channel. We define $P(t)=\bigcup_{i=1}^{N} P_{i}(t)$ as a collective region occupied by the particles at time $t$. The fluid domain is denoted by $\Omega \backslash P(t)$ at time $t$ with $\Omega$ being the entire channel. In the following sections, the governing equations and boundary conditions for both magnetic and flow problems will be discussed in detail.

\section{B. Magnetostatic problem}

The problem of electromagnetic analysis is actually a problem of solving a set of Maxwell's equations subjected to given boundary conditions. ${ }^{25}$ In the case of magnetostatics without free current, the governing equations can be written as

$$
\begin{gathered}
\nabla \times \boldsymbol{H}=\mathbf{0}, \\
\nabla \cdot \boldsymbol{B}=0,
\end{gathered}
$$

where $\boldsymbol{H}$ is the magnetic field intensity and $\boldsymbol{B}$ the magnetic flux density. To solve the two Maxwell's equations for magnetostatics, one needs a constitutive equation relating the two field quantities, $\boldsymbol{B}$ and $\boldsymbol{H}$. The constitutive equation describing macroscopic properties of the medium being considered is $\boldsymbol{B}=\mu \boldsymbol{H}$, where $\mu$ denotes the magnetic permeability of a linear isotropic medium. 
The magnetic permeability $\mu$, which is discontinuous at fluid-particle interfaces, is evaluated using a scalar function $\delta$, given by

$$
\mu(\delta)=\left\{\begin{array}{ll}
\mu_{\mathrm{p}}, & \text { if } \delta \leq 0 \text { (particle domain) } \\
\mu_{\mathrm{f}}, & \text { otherwise (fluid domain) }
\end{array},\right.
$$

where $\delta$ is the signed distance function measured from a spatial position $\boldsymbol{x}$ to the particle boundaries $\partial P_{i}(t)$, defined by

$$
\delta(\boldsymbol{x})=\min \left\{\left|\boldsymbol{x}-\boldsymbol{X}_{\boldsymbol{i}}\right|-R_{i}\right\} \quad(i=1, \cdots, N),
$$

where $\boldsymbol{X}_{i}$ is the position vector of the center of the $i$ th particle and $R_{i}$ the radius of the particle.

To solve the two governing equations (Eqs. (1) and (2)), the two equations are converted to a second-order partial differential equation involving only one field variable called the magnetic scalar potential $\phi$. The magnetic field intensity is defined by $\boldsymbol{H}=-\nabla \phi$, which satisfies Eq. (1). Therefore, the magnetic field governed by Eqs. (1) and (2) is represented by the following equation:

$$
\nabla \cdot(\mu \nabla \phi)=0
$$

subject to the continuity conditions at fluid-particle interfaces, given by

$$
\begin{gathered}
\phi_{\mathrm{f}}=\phi_{\mathrm{p}}, \\
\mu_{\mathrm{f}} \nabla \phi_{\mathrm{f}} \cdot \boldsymbol{n}=\mu_{\mathrm{p}} \nabla \phi_{\mathrm{p}} \cdot \boldsymbol{n},
\end{gathered}
$$

where $\boldsymbol{n}$ is the unit normal vector at the interfaces, and the subscript " $\mathrm{f}$ " and " $\mathrm{p}$ " refer to the potential evaluated at the fluid and particle domain, respectively. To apply a uniform magnetic field directed vertically, a constant potential difference $\Delta \phi$ between the top $\left(\Gamma_{3}\right)$ and bottom $\left(\Gamma_{1}\right)$ is imposed, such that $H_{0}=\Delta \phi / 2 h$, where $H_{0}$ is the magnitude of the external field and $2 h$ the height of the channel (see Fig. 1). The constraints for the potential difference are given by

$$
\begin{gathered}
\phi(x,-h)=\phi(x, h)+\Delta \phi, \quad \text { on } \Gamma_{1} \cup \Gamma_{3}, \\
\phi(-w, y)=\phi(w, y), \quad \text { on } \Gamma_{2} \cup \Gamma_{4},
\end{gathered}
$$

which will be treated via Lagrange multipliers in the finite element formulation. In this manner, we are able to apply an external field, which is directed vertically and periodic in the horizontal direction. To make the problem complete, a reference potential, say $\phi=0$, is specified at one point in the domain, $\Gamma_{p}$.

Given a uniform field applied externally, a non-uniform magnetic field created due to the difference in the magnetic permeability between the particle and the fluid is the source of the magnetic forces acting on the particles. To describe the forces induced by the external magnetic field and the magnetic interactions among the particles, we use a formulation based on the Maxwell stress tensor defined by $\boldsymbol{T}_{m}=\mu\left(\boldsymbol{H} \boldsymbol{H}-\frac{1}{2} H^{2} \boldsymbol{I}\right)$, where $\boldsymbol{I}$ is the identity tensor and $H^{2}=\boldsymbol{H} \cdot \boldsymbol{H}$. The body force $\boldsymbol{f}_{m}$ working on materials within the magnetic field is represented by the divergence of the Maxwell stress tensor, i.e., $\boldsymbol{f}_{m}=\nabla \cdot \boldsymbol{T}_{m}$, contributing to the momentum balance equation for the flow as an additional body force. ${ }^{19}$ In principle, there is no approximation on the magnetic force and this model can be extended to particles with arbitrary shape or particles with non-uniform magnetic properties.

\section{Flow problem}

The fluid flow is governed by the Stokes equations and the inertia of particles is neglected because of the low Reynolds number, which are assumptions valid in most microscale flows with a particle diameter of $1-10 \mu \mathrm{m} \cdot{ }^{3,7,8}$ The set of equations describing the fluid flow in $\Omega \backslash P(t)$ is given by

$$
\nabla \cdot \sigma+\nabla \cdot \boldsymbol{T}_{m}=0 \quad \text { in } \Omega \backslash P(t),
$$




$$
\begin{array}{ll}
\boldsymbol{\nabla} \cdot \boldsymbol{u}=0 & \text { in } \Omega \backslash P(t), \\
\boldsymbol{\sigma}=-p \boldsymbol{I}+2 \eta \boldsymbol{D} & \text { in } \Omega \backslash P(t), \\
\boldsymbol{u}=\boldsymbol{U}_{i}+\boldsymbol{\omega}_{i} \times\left(\boldsymbol{x}-\boldsymbol{X}_{i}\right) & \text { on } \partial P_{i}(t) \quad(i=1, \ldots, N), \\
\boldsymbol{u}=-\dot{\gamma} h & \text { on } \Gamma_{1}, \\
\boldsymbol{u}=\dot{\gamma} h & \text { on } \Gamma_{3},
\end{array}
$$

where $\boldsymbol{\sigma}$ is the Cauchy stress tensor, $\boldsymbol{u}$ the velocity, $p$ the pressure, $\eta$ the viscosity, $\boldsymbol{D}$ the rate-ofdeformation tensor, $\boldsymbol{U}_{i}$ the translational velocity of the $i$ th particle, $\boldsymbol{\omega}_{i}$ the angular velocity of the $i$ th particle, $\boldsymbol{x}$ the position vector of a point on the $i$ th particle boundary, $\boldsymbol{X}_{i}$ the position vector of the center of the $i$ th particle, and $\dot{\gamma}$ the shear rate. Equations (10)-(12) are the momentum balance equation, the continuity equation, and the constitutive relation for the fluid domain, respectively. Equations (13), (14), and (15) are the constraints for rigid body motion of the particles and the essential boundary conditions at the bottom and top boundaries, respectively. The relations for the horizontal periodicity between $\Gamma_{2}$ and $\Gamma_{4}$ are represented by

$$
\begin{array}{lr}
\boldsymbol{u}(-w, y)=\boldsymbol{u}(w, y) & \text { on } \Gamma_{2} \cup \Gamma_{4}, \\
\boldsymbol{t}(-w, y)=-\boldsymbol{t}(w, y) & \text { on } \Gamma_{2} \cup \Gamma_{4} .
\end{array}
$$

In Eq. (17), $\boldsymbol{t}$ is the traction defined by $\boldsymbol{t}=\boldsymbol{\sigma} \cdot \boldsymbol{n}$, where $\boldsymbol{n}$ is the outward unit normal vector at the boundary.

As for the particle domain $P(t)$, a rigid-ring description ${ }^{26}$ is employed. In this model, the fluid also fills the particles and the rigid body motion is imposed on the particle boundaries only. This description enables us to solve the same governing equations for both (fluid and particle) domains, reducing the number of unknowns for the rigid body constraints. The governing equations for a particle domain $P_{i}(t)$ at time $t$ are the same as Eqs. (10)-(13) for the fluid domain. Note that the rigid-ring description is valid if inertia is negligible and the particle susceptibility is constant. If we solve a problem with inertia or particles with non-uniform magnetic properties, rigid body constraints should be imposed inside the particle domain as well.

In addition to the above-mentioned equations, one needs to solve the kinematic equations for the evolution of particle positions with time $t$, represented by

$$
\begin{aligned}
\frac{d \boldsymbol{X}_{i}}{d t} & =\boldsymbol{U}_{i}, \quad \boldsymbol{X}_{i}(0)=\boldsymbol{X}_{i, 0}, \\
\frac{d \boldsymbol{\Theta}_{i}}{d t} & =\omega_{i}, \quad \boldsymbol{\Theta}_{i}(0)=\boldsymbol{\Theta}_{i, 0} .
\end{aligned}
$$

Finally, balance equations are needed for force and torque working on particles to determine the unknown rigid body motions $\left(\boldsymbol{U}_{i}, \boldsymbol{\omega}_{i}\right)$ of the particles. In the absence of inertia, the two balance equations are represented as follows:

$$
\begin{aligned}
& \boldsymbol{F}_{i}=\int_{\partial P_{i}^{+}(t)}\left(\boldsymbol{\sigma}+\boldsymbol{T}_{m}\right) \cdot \boldsymbol{n} d S=\mathbf{0}, \\
& \boldsymbol{T}_{i}=\int_{\partial P_{i}^{+}(t)} \boldsymbol{r}_{i} \times\left[\left(\boldsymbol{\sigma}+\boldsymbol{T}_{m}\right) \cdot \boldsymbol{n}\right] d S=\mathbf{0},
\end{aligned}
$$

where $\partial P_{i}^{+}$is the outer boundary of the $i$ th particle, $\boldsymbol{F}_{i}$ the sum of the hydrodynamic and magnetic forces, and $\boldsymbol{T}_{i}$ the sum of the hydrodynamic and magnetic torques working on a rigid particle $P_{i}$ at the time $t$. 


\section{Scaling and Mason number}

To obtain a non-dimensional form of the momentum equation (Eq. (10)), non-dimensional variables (with a superscript*) are defined by

$$
\begin{aligned}
x^{*} & =\frac{x}{l_{c}}, \quad y^{*}=\frac{y}{l_{c}}, \\
\boldsymbol{u}^{*} & =\frac{\boldsymbol{u}}{u_{c}}, \\
p^{*} & =\frac{p}{p_{c}}, \\
T_{i j}^{*} & =\frac{T_{i j}}{\mu_{0} H_{c}^{2}},
\end{aligned}
$$

where $l_{c}$ denotes a characteristic length, $\boldsymbol{u}_{c}$ a characteristic velocity, $p_{c}$ a characteristic pressure, $\mu_{0}$ the magnetic permeability in vacuum, and $H_{c}$ the characteristic magnetic field intensity. Here, we define the characteristic length as a particle radius, i.e., $l_{c}=a$. The characteristic time is defined as the inverse of the shear rate, $t_{c}=\dot{\gamma}^{-1}$, thus $t^{*}=\dot{\gamma} t$. Then, the characteristic velocity and pressure are defined as $u_{c}=a \dot{\gamma}$ and $p_{c}=\eta u_{c} / a=\eta \dot{\gamma}$, respectively. The Maxwell stress tensor $T_{i j}$ is nondimensionalized by $\mu_{0} H_{c}^{2}$. The characteristic magnetic field intensity $H_{c}$ is defined as $H_{c}=\beta H_{0}$, where $H_{0}$ is the magnitude of the external field and $\beta$ is the effective polarization factor. The factor $\beta$ is adopted from the measure of effective polarization in the case of a spherical particle in a uniform field as a function of $\mu_{\mathrm{p}}$ and $\mu_{\mathrm{f}},{ }^{24}$ defined by

$$
\beta=\frac{\mu_{\mathrm{p}}-\mu_{\mathrm{f}}}{\mu_{\mathrm{p}}+2 \mu_{\mathrm{f}}}=\frac{\chi_{\mathrm{p}}}{3+\chi_{\mathrm{p}}},
$$

where $\chi_{p}$ is the magnetic susceptibility of the particles. Here, since the fluid is assumed to be non-magnetic, $\mu_{\mathrm{f}}=\mu_{0}$. The magnetic permeability of particle is related to the susceptibility by $\mu_{\mathrm{p}}=\mu_{0}\left(1+\chi_{\mathrm{p}}\right)$.

Then, the resulting non-dimensional momentum equation becomes

$$
\nabla^{*} p^{*}-\nabla^{* 2} \boldsymbol{u}^{*}=\frac{1}{\mathrm{Mn}} \nabla^{*} \cdot \boldsymbol{T}^{*}
$$

where $\mathrm{Mn}$ is the Mason number, the ratio of viscous force to magnetic force, defined by

$$
\mathrm{Mn}=\frac{\eta \dot{\gamma}}{\mu_{0} \beta^{2} H_{0}^{2}} .
$$

The Mason number can also be regarded as the ratio of the magnetic time scale $t_{m}=\eta /\left(\mu_{0} \beta^{2} H_{0}^{2}\right)$ and the flow time scale $t_{f}=\dot{\gamma}^{-1}$. At a lower Mason number, magnetic forces are dominant, while at a higher Mason number, viscous forces are dominant. In addition to the Mason number, particle fraction and the difference in the magnetic permeability between the fluid and the particles are also known as parameters with influences on the dynamics of magnetic particles. ${ }^{17,18}$ In simulations introduced in the following sections, we mainly focus on the effect of the Mason number and the particle fraction on the particle motion and the fluid flow.

\section{NUMERICAL METHODS}

To solve the flow with suspended paramagnetic particles under the influence of an external magnetic field, the numerical methods we developed previously ${ }^{19}$ are used. In the following sections, we briefly introduce the finite element formulations to solve the magnetic and flow problems for the given boundary conditions and constraints. For other details, such as the validation of the method and the comparison with other methods, we refer to our previous publications. ${ }^{8,19}$ 


\section{A. Magnetic problem}

The weak form of the Poisson equation (Eq. (5)) and constraints (Eqs. (8) and (8)) for the magnetic potential $\phi$ is given as: find the magnetic potential $\phi$ in $\mathbb{S}$ such that

$$
\begin{aligned}
& \int_{\Omega} \mu \nabla \phi \cdot \nabla \psi d A+\left\langle\lambda^{m, v}, \psi(x,-h)-\psi(x, h)\right\rangle_{\Gamma_{1}} \\
& +\left\langle\lambda^{m, h}, \psi(-w, y)-\psi(w, y)\right\rangle_{\Gamma_{4}}=0, \\
& \left\langle\mu^{m, v}, \phi(x,-h)-\phi(x, h)\right\rangle_{\Gamma_{1}}=\left\langle\mu^{m, v}, \Delta \phi\right\rangle_{\Gamma_{1}}, \\
& \left\langle\mu^{m, h}, \phi(-w, y)-\phi(w, y)\right\rangle_{\Gamma_{4}}=0,
\end{aligned}
$$

for all $\psi \in \mathbb{S}_{0}$ with $\mathbb{S}=\left\{\phi \in H^{1}(\Omega)\right.$ with $\phi=\bar{\phi}$ at $\left.\Gamma_{p}\right\}$ and $\mathbb{S}_{0}=\left\{\psi \in H^{1}(\Omega)\right.$ with $\psi=0$ at $\left.\Gamma_{p}\right\}$. In the weak formulation, two constraints for the potential difference in the vertical and horizontal direction are treated via the Lagrange multipliers, $\lambda^{m, v}$ and $\lambda^{m, h}$, defined on the boundaries, $\Gamma_{1}$ and $\Gamma_{4}$, respectively. The continuity of the potential $\phi$, Eq. (6), holds in the standard finite element formulation employing a continuous interpolation function. The continuity of the normal component of $\boldsymbol{B}$, Eq. (7), which is represented by the continuity of flux in the diffusion equation of the magnetic potential $\phi$, is weakly satisfied in the finite element formulation. The weak form is used to obtain an approximate solution using the finite element method with bi-quadratic interpolation for $\phi$ and linear interpolations for the Lagrange multipliers. In the numerical integration of the weak form, the permeability $\mu$ is evaluated by Eq. (3) using the distance function $\delta$ at each integration point. The resulting matrix equation is solved using a sparse Gauss elimination method to solve a symmetric matrix, HSL/MA57. ${ }^{27}$

\section{B. Flow problem}

The fluid flow problem, including interactions between fluid and rigid particles, is solved by using a fictitious domain method and the finite element method. We use a fictitious domain method to treat rigid body constraints on particle boundaries. In the derivation of the weak form, following the approach of Glowinski et al. ${ }^{23}$ fluid-particle interactions are implicitly treated through the combined weak formulation, where hydrodynamic force and torque on the particle boundaries are canceled exactly. In the combined weak formulation, the rigid body constraint is enforced by the constraint equation using Lagrange multipliers, $\lambda^{\mathrm{p}, i}$, defined on the particle boundary $\partial P_{i}(i=1, \ldots, N)$.

The space for the combined velocity, $\mathbb{V}$, is defined by

$$
\begin{aligned}
\mathbb{V}= & \left\{\left(\boldsymbol{u}, \boldsymbol{U}_{i}, \boldsymbol{\omega}_{i}\right) \mid \boldsymbol{u} \in H^{1}(\Omega)^{2}, \boldsymbol{U}_{i} \in \mathbb{R}^{2}, \boldsymbol{\omega}_{i} \in \mathbb{R},\right. \\
& \left.\boldsymbol{u}=\boldsymbol{U}_{i}+\boldsymbol{\omega}_{i} \times \boldsymbol{r}_{i} \text { on } \partial P_{i}(t) \text { and } \boldsymbol{u}=\mathbf{0} \text { on } \Gamma_{w}\right\}
\end{aligned}
$$

for $i=1, \ldots, N$. For a given particle configuration $\boldsymbol{X}_{i}(i=1, \ldots, N)$, the weak form for the entire domain $\Omega$ can be stated as follows: find $\left(\boldsymbol{u}, \boldsymbol{U}_{i}, \boldsymbol{\omega}_{i}\right) \in \mathbb{V}, p \in L^{2}(\Omega)$, and $\lambda^{\mathrm{p}, i} \in L^{2}\left(\partial P_{i}(t)\right)$ $(i=1, \ldots, N)$ such that

$$
\begin{aligned}
\int_{\Omega} & 2 \eta \boldsymbol{D}(\boldsymbol{v}): \boldsymbol{D}(\boldsymbol{u}) d A-\int_{\Omega}(\nabla \cdot \boldsymbol{v}) p d A \\
& +\sum_{i=1}^{N}\left\langle\boldsymbol{v}-\left(\boldsymbol{V}_{i}+\chi_{i} \times \boldsymbol{r}_{i}\right), \lambda^{\mathrm{p}, i}\right\rangle_{\partial P_{i}} \\
& +\left\langle\lambda^{\mathrm{h}}, \boldsymbol{v}(-w, y)-\boldsymbol{v}(w, y)\right\rangle_{\Gamma_{4}}=-\int_{\Omega} \boldsymbol{D}(\boldsymbol{v}): \boldsymbol{T}_{\mathrm{m}} d A, \\
& -\int_{\Omega} q(\nabla \cdot \boldsymbol{u}) d A=0,
\end{aligned}
$$




$$
\begin{gathered}
\left\langle\boldsymbol{\mu}^{\mathrm{p}, i}(\boldsymbol{x}), \boldsymbol{u}(\boldsymbol{x})-\left(\boldsymbol{U}_{i}+\boldsymbol{\omega}_{i} \times \boldsymbol{r}_{i}\right)\right\rangle_{\partial P_{i}}=0, \\
\left\langle\boldsymbol{\mu}^{\mathrm{h}}, \boldsymbol{u}(-w, y)-\boldsymbol{u}(w, y)\right\rangle_{\Gamma_{4}}=0,
\end{gathered}
$$

for all $\left(\boldsymbol{v}, \boldsymbol{V}_{i}, \chi_{i}\right) \in \mathbb{V}, q \in L^{2}(\Omega), \boldsymbol{\mu}^{\mathrm{p}, i} \in L^{2}\left(\partial P_{i}(t)\right)(i=1, \ldots, N)$, and $\boldsymbol{\mu}^{\mathrm{h}} \in L^{2}\left(\Gamma_{4}\right)$. To treat the body force in the weak formulation, instead of using the divergence of the Maxwell stress tensor as it is, integration by part and the Gauss theorem are applied to circumvent differentiation of the Maxwell stress tensor, which is discontinuous at fluid-particle interfaces. We use bi-quadratic interpolation for the velocity and bi-linear interpolation for the pressure, the so-called $Q_{2} / Q_{1}$ element. Bi-linear interpolation is used to discretize the Lagrange multipliers. The resulting matrix equation is solved by the same direct sparse matrix solver as used in the magnetic problem.

Particle positions are updated by integrating the evolution equations, Eq. (18), using the rigid body motions of the particles obtained as a part of the solution. We employ explicit time integration schemes. The explicit Euler method (Eq. (37)) is used at the first time step and the second-order Adams-Bashforth method (Eq. (38)) from the second time step onward:

$$
\begin{aligned}
& \boldsymbol{X}_{i}^{n+1}=\boldsymbol{X}_{i}^{n}+\Delta t \boldsymbol{U}_{i}^{n}, \\
& \boldsymbol{X}_{i}^{n+1}=\boldsymbol{X}_{i}^{n}+\Delta t\left(\frac{3}{2} \boldsymbol{U}_{i}^{n}-\frac{1}{2} \boldsymbol{U}_{i}^{n-1}\right),
\end{aligned}
$$

where variables with superscripts $n-1, n$, and $n+1$ represent those evaluated at the previous time, the present time, and the next time step, respectively.

Based on the particle speed $U_{i}$ and the mesh size $h_{e}$, the time step $\Delta t$ is chosen to be less than both the convection time scale of the particles $t_{p, i}=h_{e} / U_{i}$ and the magnetic time scale $t_{m}=\eta /\left(\mu_{0} \beta^{2} H_{0}^{2}\right)$, i.e., $\Delta t<\min \left(t_{p, i}, t_{m}\right), i=1, \ldots, N$. The time step chosen in this manner is sufficient enough to obtain smooth particle motions. If the selected time step does not satisfy the criterion, the chain motion shows unrealistic oscillation and the angular velocity of each particle is not smooth, but shows spurious fluctuation in time.

\section{Remarks on the numerical scheme}

The direct numerical simulation method is capable of taking into account both hydrodynamic and magnetic interactions in a coupled manner. ${ }^{19}$ As for the magnetic forces, the point-dipole approximation is simple and quite effective if the length scale of the field non-uniformity is much larger than the particle size. ${ }^{24,28}$ However, this assumption may be violated in applications with the particle size comparable to the length scale of the field non-uniformity due to closely packed particles forming clusters or chains. In the present approach, the magnetic interactions are computed from the solution of a magnetic potential problem for the entire domain including both the particles and the fluid via a Maxwell stress formulation, which is believed to be the most general way to treat the magnetic forces in magnetic particulate flows. The numerical method captures both longand short-range hydrodynamic interactions among particles using a fictitious domain method in the combined finite element formulation. In addition, the present scheme has several other advantages over particle-based numerical methods: (1) easy implementation of particles with arbitrary shape in a code based on the finite element method; (2) easy treatment of inhomogeneous magnetic permeability, possibly also depending on the magnetic field intensity; (3) direct simulation of nonNewtonian flows with magnetic particles, for example, functionalized microbeads suspended in a body fluid applicable to diagnostic lab-on-a-chip devices. But, our numerical scheme requires much more computational resources than particle-based numerical methods used more frequently.

\section{RESULTS AND DISCUSSION}

In the simulations, we use a periodic channel with $w / h=1$. The shear rate $\dot{\gamma}$ is fixed to 1 . The origin of the coordinate system is located at the center of the square channel. The channel geometry 
is discretized into 40000 nine-node quadrilateral elements. The ratio of particle radius to the channel height $a / 2 h$ is 0.05 . The size of line elements describing the particle boundary is determined such that the size is comparable to the size of the fluid mesh.

\section{A. Single-chain problem in the shear flow}

We begin with a two-particle problem, where a chain consisting of two paramagnetic particles is initially located at the center of the square channel, aligned to the magnetic field directed vertically. The non-dimensional position of a particle is defined by $\left(X^{*}, Y^{*}\right)$, where $X^{*}=X / a$ and $Y^{*}=Y / a$. The initial non-dimensional positions of the two particles, $P_{1}$ and $P_{2}$, are $(0,-1)$ and $(0,1)$, respectively. Once the drag velocity is imposed, the chain rotates clockwise, as schematically depicted in Fig. 2. The angle of rotation $\theta$ at an instance is defined by the angle measured from the field direction to the line connecting the centers of the two particles.

The single-chain problem with a magnetic susceptibility $\chi_{\mathrm{p}}=1$ is solved first. Here, we are mainly concerned with the effect of the Mason number on the rotational dynamics of the chain. Figure 3 shows the change of $\theta$ with the non-dimensional time $t^{*}(=\dot{\gamma} t)$ at several Mason numbers. At lower Mason numbers up to $\mathrm{Mn}=0.32$, there exists an equilibrium, where the two torques working on the chain, the magnetic torque due to the external field and the viscous torque due to the shear flow, are in balance. Starting from an initial location, the chain rotates and reaches a stationary configuration, resulting in an equilibrium tilting angle $\theta_{\mathrm{eq}}$. In the low Mason number regime, the equilibrium angle at each Mason number is found to increase linearly with the Mason number. Above a critical Mason number $\left(\mathrm{Mn}_{c}=0.32\right)$, however, the magnetic torque acting on the chain can no longer resist the chain rotation, resulting in a continuous rotation of the chain. At critical Mason number, the angle of rotation is approximately $45^{\circ}$, which is the maximum rotation angle attainable in this case.

Depicted in Fig. 4 are the trajectories of the two particles with time at two Mason numbers, $\mathrm{Mn}=0.32$ and $\mathrm{Mn}=1.6$, as representative examples showing two distinct behaviors in the particle motion: one reaching equilibrium (Fig. 4(a)), where the particles are at rest, and the other exhibiting periodic motion (Fig. 4(b)), depending on the Mason number. Figure 5 shows the change of the

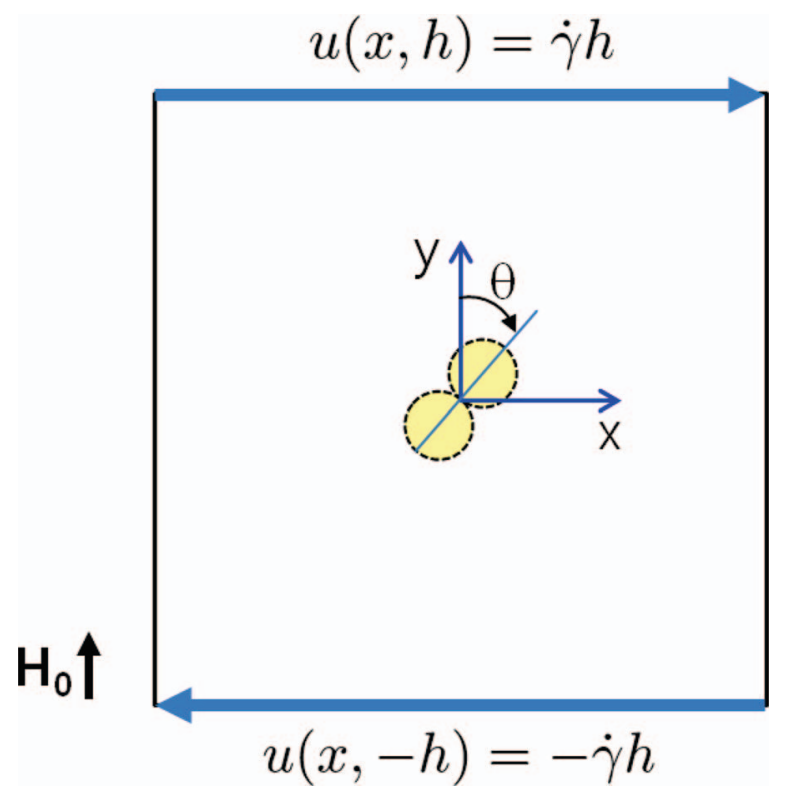

FIG. 2. Rotation of two magnetic particles suspended in a shear flow under the influence of an externally applied magnetic field $\boldsymbol{H}_{0}$ directed vertically. The chain of two particles is initially aligned with the field direction and rotates due to continuous shearing. Here, $\theta$ is the rotating angle measured from the $y$-axis to the line connecting the centers of two particles at an instance. 


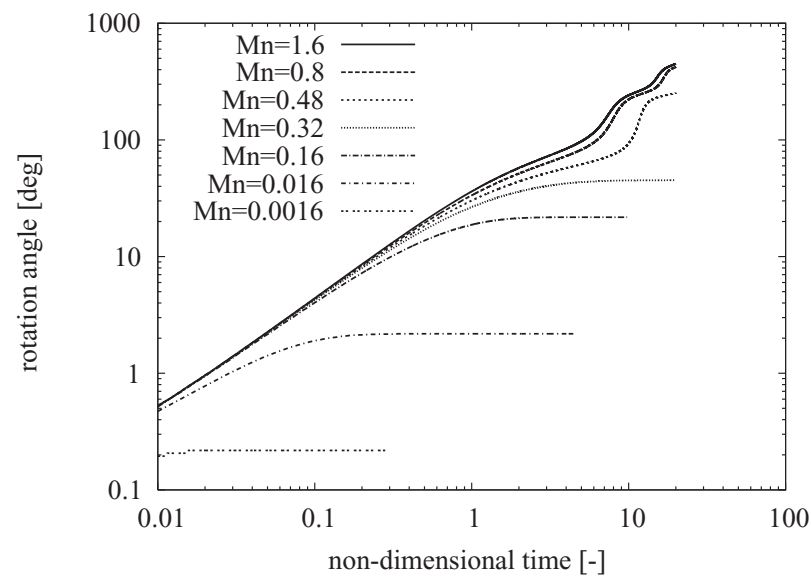

FIG. 3. Rotation angle $\theta$ (defined in Fig. 2) with the non-dimensional time $t^{*}=\dot{\gamma} t$ at several Mason numbers. The magnetic susceptibility of the particles $\chi_{\mathrm{p}}$ is fixed to 1 .

non-dimensional angular velocity $\Omega_{z}^{*}\left(=\Omega_{z} / \dot{\gamma}\right)$ of the first particle $\left(P_{1}\right)$ about its center as a function of the non-dimensional time $t^{*}$. In this two-particle case, the rotational behavior of the second particle $\left(P_{2}\right)$ is the same as that of the first one $\left(P_{1}\right)$. It is found that the rotation rate of each particle about its center is the same as that of the chain of two particles, i.e., the two particles move like a single rigid body and there is no relative rolling between the two particles. At lower Mason numbers, $\mathrm{Mn}<\mathrm{Mn}_{\mathrm{c}}$, where there is an equilibrium configuration of the chain, the angular velocity of each particle approaches zero. In a higher Mason number regime, $\mathrm{Mn}>\mathrm{Mn}_{\mathrm{c}}$, however, the rotational motion of the particles is time periodic. From Figs. 4 and 5, we can conclude that, at the higher Mason numbers above $\mathrm{Mn}_{c}$, the motion of each particle is periodic in time and the velocity field is consequently time periodic as well. In this regime, the larger the Mason number, the shorter the time period.

Finally, the effect of the magnetic susceptibility on the rotational dynamics is discussed. Shown in Fig. 6 is the change of the angle of rotation at the two Mason numbers, $\mathrm{Mn}=0.016$ and $\mathrm{Mn}=0.16$, where $\mathrm{Mn}<\mathrm{Mn}_{\mathrm{c}}$. At a fixed Mason number, the higher the susceptibility, the larger the rotation angle $\theta_{\text {eq }}$, implying that, in addition to the Mason number defined in Eq. (28), the susceptibility is also a parameter governing chain dynamics. Therefore, the difference in magnetic property between the particle and the fluid (in this study characterized by the magnetic susceptibility $\chi_{\mathrm{p}}$ ) also has an influence on the dynamics of the particles. In the low Mason number regime, as depicted in Fig. 7, the equilibrium angle $\theta_{\text {eq }}$ increases linearly with the Mason number at a fixed susceptibility.

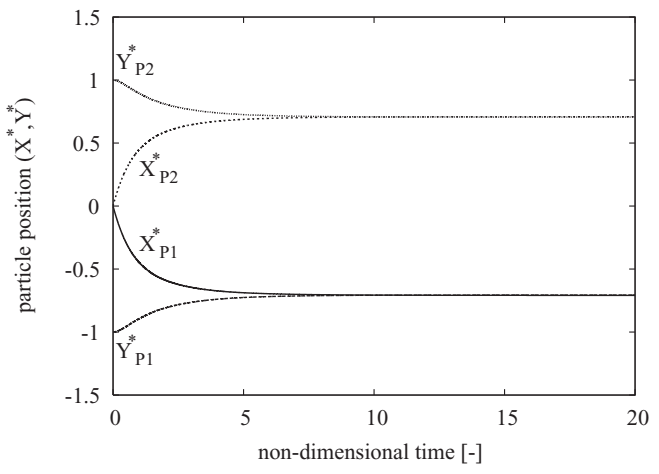

(a)

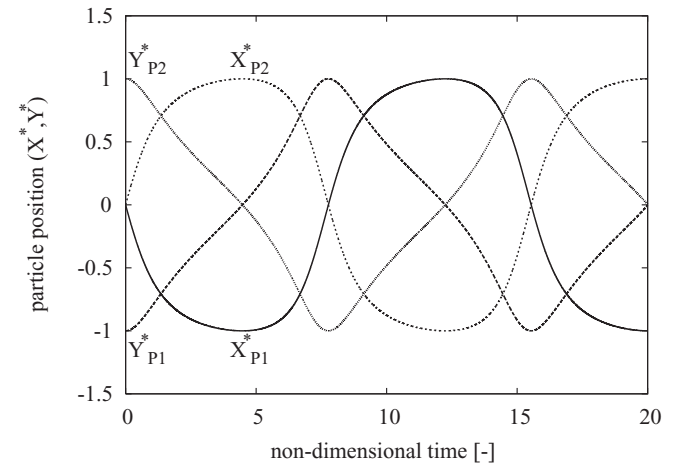

(b)

FIG. 4. Trajectory of the two particles at the two Mason numbers, (a) $\mathrm{Mn}=0.32$ and (b) $\mathrm{Mn}=1.6$, with the susceptibility $\chi_{\mathrm{p}}=1$. 


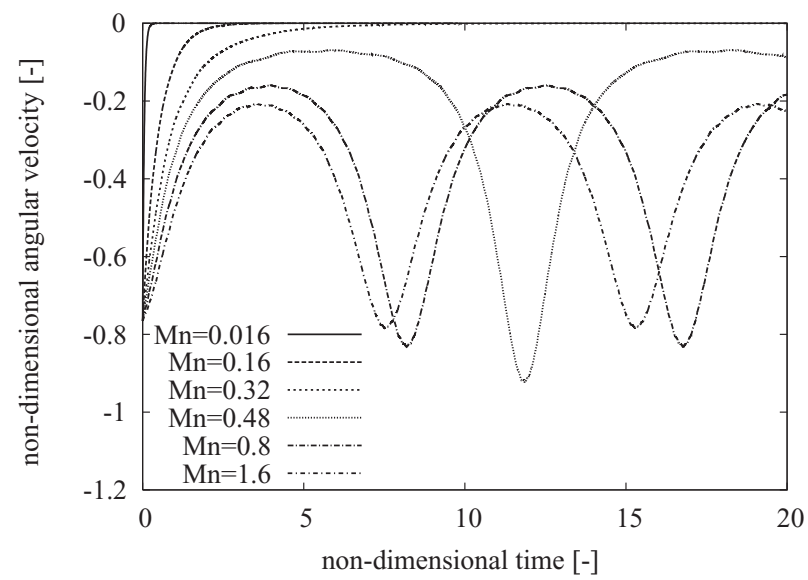

FIG. 5. Non-dimensional angular velocity $\Omega_{z}^{*}$ of the particle $P_{1}$ with the non-dimensional time $t^{*}=\dot{\gamma} t$ at several Mason numbers with $\chi_{\mathrm{p}}=1$.

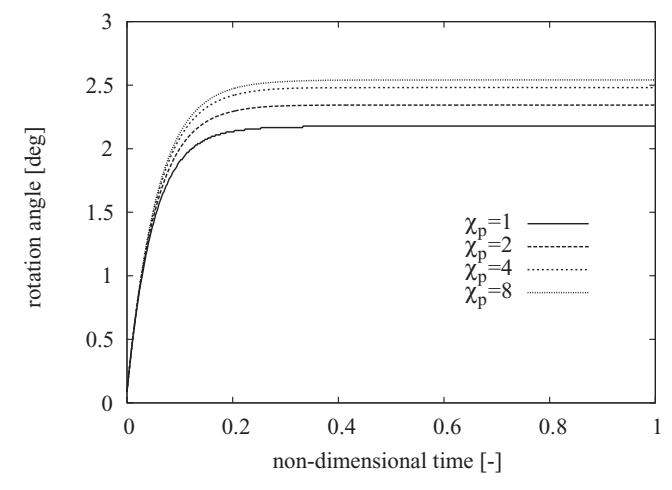

(a)

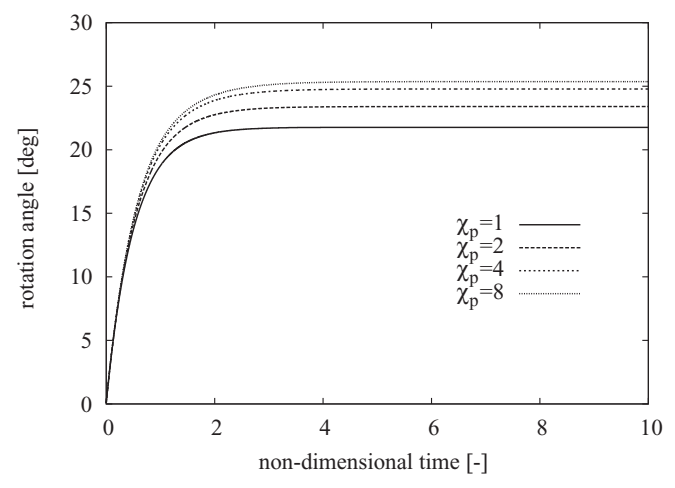

(b)

FIG. 6. Change of the rotation angle dependent on the magnetic susceptibility at (a) $\mathrm{Mn}=0.016$ and (b) $\mathrm{Mn}=0.16$.

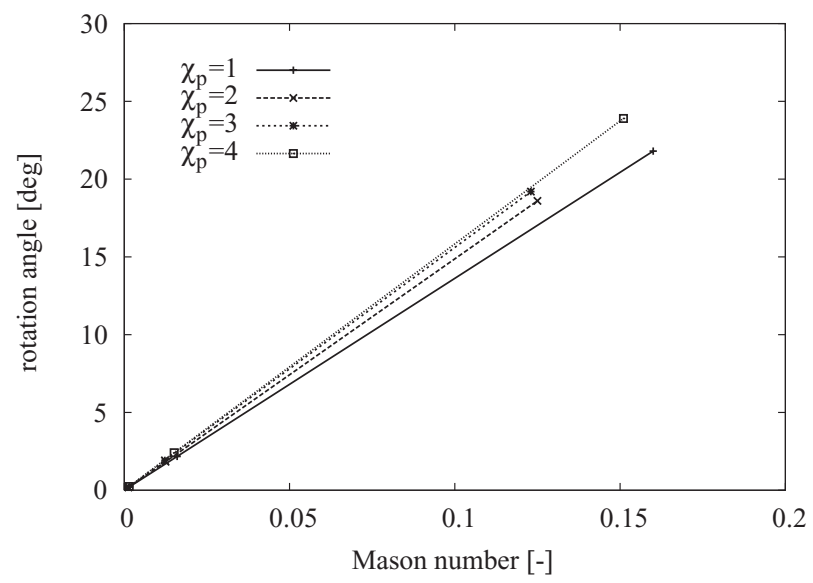

FIG. 7. Equilibrium angle of rotation as a function of the Mason number, dependent on the susceptibility $\chi_{p}$. 


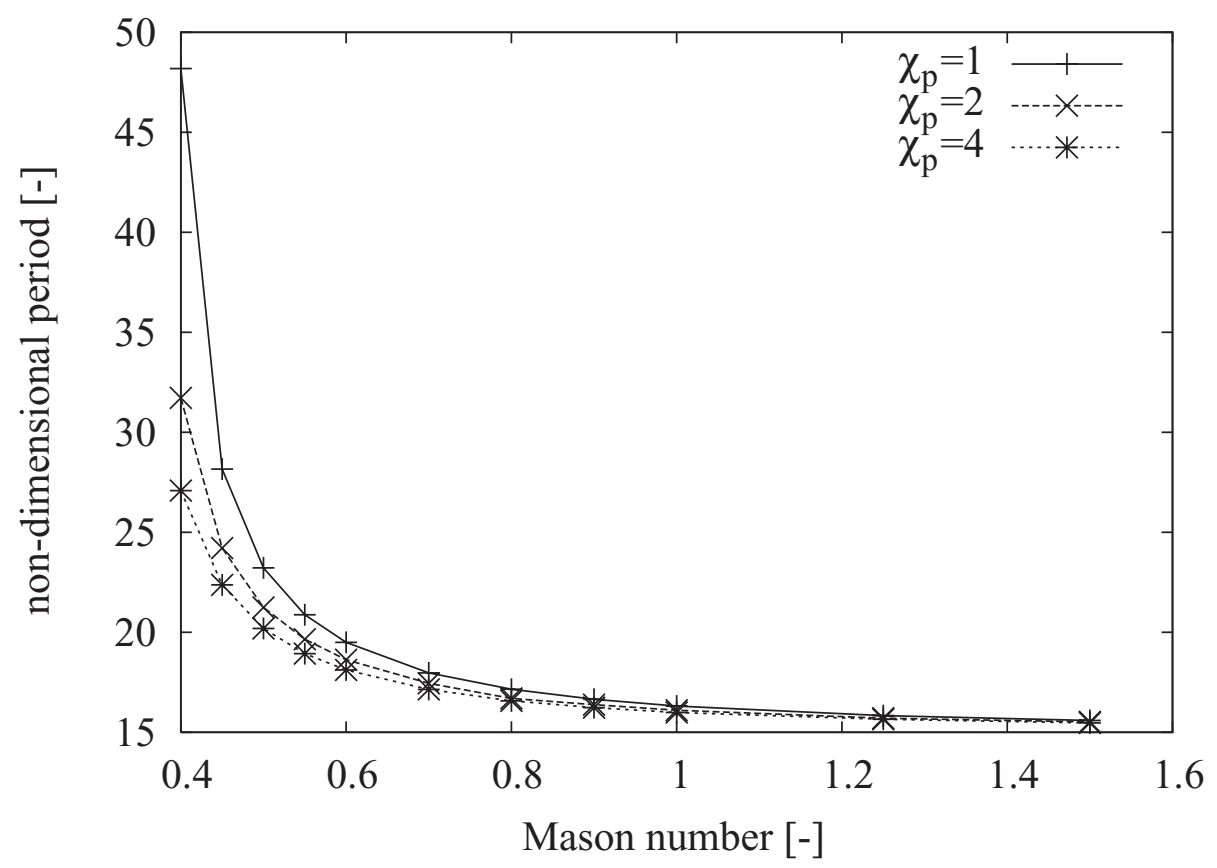

FIG. 8. Non-dimensional period of rotational motion $T_{p}^{*}=T_{p} \dot{\gamma}$ as a function of the Mason number, dependent on the susceptibility $\chi_{\mathrm{p}}$. Here, $T_{p}$ is the time period and $\dot{\gamma}$ is the shear rate applied.

As the equilibrium angle is affected by the Mason number and the susceptibility, one can conjecture that the period $T_{p}$ of the particle motion above a critical Mason number will also be influenced by those parameters. Figure 8 shows the non-dimensional period $T_{p}^{*}$ of chain rotation, defined by $T_{p}^{*}=T_{p} \dot{\gamma}$, as a function of the Mason number at three values of the susceptibility. As the Mason number approaches the critical Mason number at a fixed susceptibility, the period increases rapidly. The rapid increase in the period is due to the increase in the resisting magnetic torque on the chain (directed counterclockwise) when the centers of the two particles are on the first and the third quadrants, i.e., $0^{\circ}<\theta<90^{\circ}$ or $180^{\circ}<\theta<270^{\circ}$. As the Mason number increases, however, the period approaches an asymptotic value (the period for $\mathrm{Mn}=\infty$ ). We can also conclude that the critical Mason number decreases with the susceptibility of the particles. Since the chain of two particles behaves like a rigid body, it will be interesting to compare the asymptotic value of the period with that predicted from Jeffery orbit, ${ }^{29}$ where the period for an ellipsoid with the aspect ratio $r_{e}$ is $T_{p}=2 \pi\left(r_{e}+1 / r_{e}\right) / \dot{\gamma}$. The non-dimensional period, predicted from Jeffery orbit with the aspect ratio $r_{e}=2$, is $5 \pi(\approx 15.7)$, while the period in the confined shear flow with $\mathrm{Mn}=\infty$ is 15.4.

\section{B. Flow characteristics}

In this section, change of flow characteristics in terms of the perturbed velocity $\boldsymbol{u}^{\prime}$ and the increase in the effective wall shear stress due to the external field are discussed. A single circular non-magnetic particle suspended in the shear flow with a shear rate $\dot{\gamma}$ rotates with the angular velocity $\omega=-0.5 \dot{\gamma}$ in an unbounded domain, leading to a perturbed velocity field, which is hyperbolic in nature. ${ }^{30}$ In this study, the perturbed velocity $\boldsymbol{u}^{\prime}$ is defined by $\boldsymbol{u}^{\prime}=\boldsymbol{u}_{0}-\boldsymbol{u}$, where $\boldsymbol{u}_{0}$ is the velocity of the shear flow without the particle and $\boldsymbol{u}$ is the velocity of the particulate flow in the presence of a uniform field and continuous shearing.

Figure 9 depicts streamlines obtained from the perturbed velocity $\boldsymbol{u}^{\prime}$ at the two Mason numbers with the susceptibility $\chi_{\mathrm{p}}=1$. Shown in Fig 9 (a) are the streamlines at equilibrium when $\mathrm{Mn}=0.16$. At this Mason number $\left(\mathrm{Mn}<\mathrm{Mn}_{\mathrm{c}}\right)$, the chain of two particles reaches an equilibrium configuration, where the chain is at rest and the perturbed flow around the chain of two particles is elliptic in nature. 


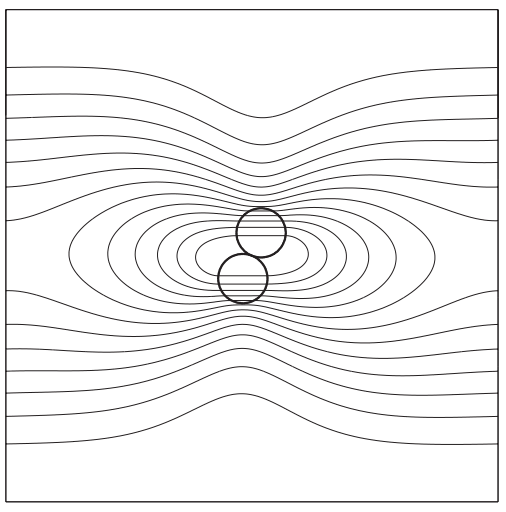

(a)

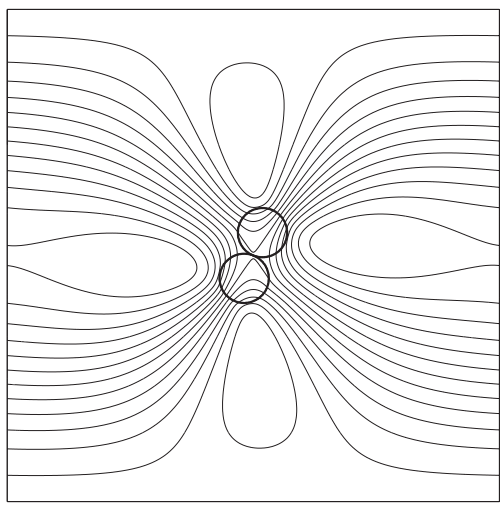

(b)

FIG. 9. Instantaneous streamlines obtained from the perturbed velocity, defined by $\boldsymbol{u}^{\prime}=\boldsymbol{u}_{0}-\boldsymbol{u}$, where $\boldsymbol{u}_{0}$ is the velocity without particle and $u$ the velocity with two suspended particles, at the two Mason numbers, (a) $\mathrm{Mn}=0.16$ and (b) $M n=\infty$. At $M n=0.16$, the two particles reach their equilibrium positions, where the perturbed flow is elliptic in nature. While, in the other case $(\mathrm{Mn}=\infty)$, where there is no external field and the particles are at the same location as in (a), the perturbed flow is hyperbolic in this shear flow.

Without magnetic field $(\mathrm{Mn}=\infty)$, however, the perturbation velocity generates hyperbolic flow around the chain (see Fig. 9(b)), similarly to the single non-magnetic particle in the shear flow.

Next, we solve a flow with chains in a uniform field to investigate the so-called magnetoviscous effect, the increase in the effective viscosity due to the field-induced structure of particles. The particle susceptibility is fixed to 1 and each chain consists of eight particles. Initially, the chains are spaced equally and aligned with the field direction. The magnetic field is perpendicular to the imposed shear flow. Similar to the two-particle problem introduced in Sec. IV A, for the given susceptibility, there is a critical Mason number, which is approximately 0.2 in this case. Below the critical number, an equilibrium between the magnetic torque and viscous torque exists. Shown in Fig. 10 are streamlines at equilibrium obtained from the velocity field with two chains at $\mathrm{Mn}=0.016$ and 0.16. In the low Mason number regime $\left(\mathrm{Mn}<\mathrm{Mn}_{\mathrm{c}}\right)$, as depicted in Fig. 10, the equilibrium angle of rotation increases with the Mason number. The chains in this regime act as barriers against the fluid flow, increasing flow resistance as the Mason number decreases. Therefore, only the regions in-between the walls and both tips of the chains play a role of the effective flow passage in the shear flow. The reduction in the effective flow passage in the lower Mason number regime, leading to higher effective viscosity, is the cause of the so-called magnetoviscous effect in this shear flow.

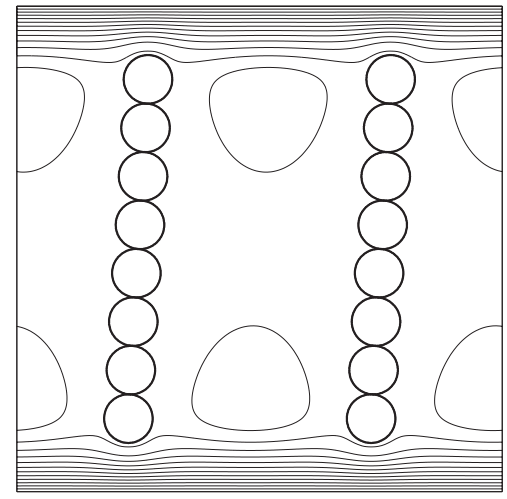

(a)

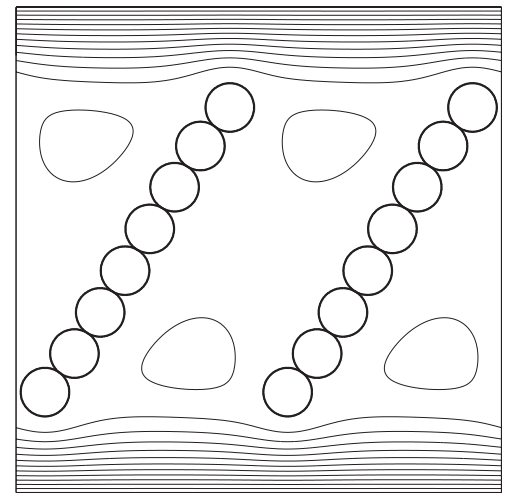

(b)

FIG. 10. Streamlines at equilibrium, where the magnetic torque and the viscous torque acting on the two chains are in balance. The particle susceptibility is $\chi_{\mathrm{p}}=1$. (a) $\mathrm{Mn}=0.016$. (b) $\mathrm{Mn}=0.16$. 


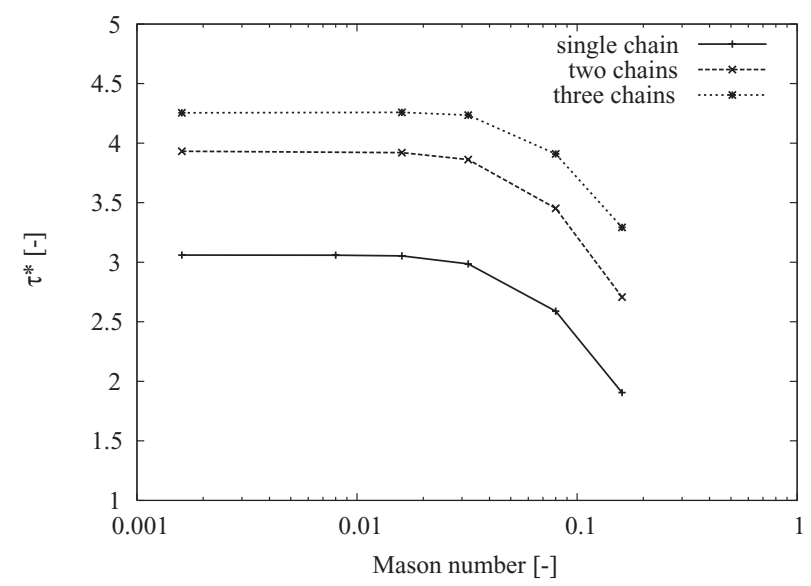

FIG. 11. Non-dimensional average wall shear stress $\tau_{w}^{*}$ as a function of the Mason number with the variation in the number of chains suspended in the channel.

In this particular case, we define the average wall shear stress $\bar{\tau}_{y x}$ as follows:

$$
\bar{\tau}_{y x}=\frac{\int_{\Gamma_{1} \cup \Gamma_{3}} \tau_{y x} d x}{L},
$$

where $L$ is the total length of the both boundaries, $\Gamma_{1}$ and $\Gamma_{3}$. Below the critical Mason number $\left(\mathrm{Mn}_{\mathrm{c}}\right)$, where there is an equilibrium tilting angle at a given Mason number, the wall shear stress also reaches a steady state. Above $\mathrm{Mn}_{\mathrm{c}}$, the wall shear stress shows oscillatory behavior in time. Shown in Fig. 11 is the variation of the non-dimensional shear stress at equilibrium, $\tau_{w}^{*}=\bar{\tau}_{y x} / \eta \dot{\gamma}$, as a function of the Mason number and the number of chains suspended in the channel. Below $\mathrm{Mn}=0.01$, the non-dimensional shear stress seems to be stationary. Above $\mathrm{Mn}=0.01$, as the Mason number increases, thinning behavior is observed, due to the increased tilting angle of the chains, resulting in wider effective flow passage. It should be noted that, as the Mason number approaches zero, the tilting angle of the chains approaches zero as well and the wall shear stress approaches an asymptotic value. Therefore, yield behavior, as reported in the literature, ${ }^{1,14}$ cannot be observed in this particular flow.

\section{Chain rupture in the shear flow}

Finally, we investigate chain rupture and reformation due to the combined effect of the shear flow and the uniform magnetic field applied externally, characterized by the Mason number. In electrorheological (ER) fluids in a DC electric field, which has an analogy with our magnetostatic case, suspensions of particles in a dielectric liquid exhibit electric field-dependent viscosity, the so-called electroviscous effect. ${ }^{11-14}$ The counterpart in magnetic suspensions is the magnetoviscous effect, ${ }^{1}$ the increase of effective viscosity with increasing magnetic field strength. According to previous works by other researchers, due to the applied shear flow and subsequent deformation, these chains tilt and stretch. Above a critical tilting angle, breaking of chains has been observed experimentally and also numerically. Three models for the electroviscous effect of ER fluids in shear flow are reported in the literature: ${ }^{11,12,24}$ (i) fractured chain remnants adhere to the electrodes (top and bottom walls in this study); (ii) shortened chains filling the channel, resisting the rotation due to the shear flow; and (iii) formation of columnar structures which deform, break, and reform during continuous shear.

Motivated by the previous works on particle chaining in ER fluids, ${ }^{11-13,15}$ we investigate further the detailed dynamics of paramagnetic particles suspended in a non-magnetic fluid. Our main concern is the effect of the Mason number and the susceptibility of the particles on fluid flow and topological changes such as tilting, breakup, and reformation of chains, which finally determine the rheological behavior of the magnetic suspension. The number of particles contained in the periodic channel is 


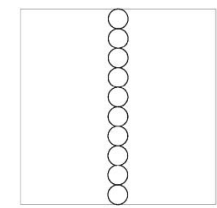

(a)

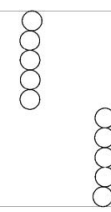

(b)

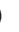

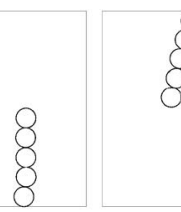

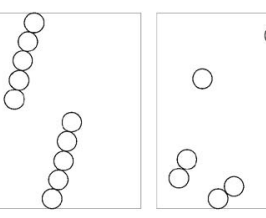

(c) (d)

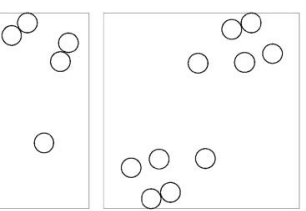

(e)

FIG. 12. Snapshots of typical chain conformations at the four Mason numbers. The area fraction of the particles is $7.85 \%$. (a) Initial configuration of a single chain consisting of 10 particles, spanning the gap between top and bottom boundaries, and typical chain conformations at (b) $\mathrm{Mn}=0.0016$, (c) $\mathrm{Mn}=0.016$, (d) $\mathrm{Mn}=0.16$, and (c) $\mathrm{Mn}=1.6$.

also varied to see the effect of the particle fraction on the topology of the chains. A confined shear flow is chosen as a model problem, where initially intact chains (spanning the channel gap) are assumed to be clinging to both top and bottom walls due to the magnetic field applied externally.

We begin with a single-chain problem, where initially the chain is aligned with the field directed vertically (see Fig. 12(a)). Up on applying continuous shearing, the chain deforms and is broken into smaller chains due to the shear flow, resulting in different structures after breakup, depending on the Mason number (see Fig. 12 showing snapshots of chain conformations). At lower Mason numbers, where $\mathrm{Mn}<0.1$ ), a chain is broken at the center of the chain with both ends cling to the wall. The detached pair of chains move in the opposite direction, form a single chain instantaneously, and the single chain is broken again. This process repeats itself. Before breakup, each chain deforms affinely until a critical tilting angle at which the chain breaks up. At higher Mason numbers (see Figs. 12(d) and 12(e)), detached chains consisting of two particles or single particles fill the channel, leading to less resistance to the flow compared with the cases with $\mathrm{Mn}<0.1$.

At the lower Mason number regime $(\mathrm{Mn}<0.1)$, the critical tilting angle $\theta_{c}$ just before breakup decreases slightly with the Mason number, from $16^{\circ}$ at $\mathrm{Mn}=0.0016$ to $15^{\circ}$ at $\mathrm{Mn}=0.016$. The predicted critical angles are smaller than predictions for an ER fluid by the point-dipole limit, where $\theta_{c}=21^{\circ}$, and an experimentally observed value around $28^{\circ} .{ }^{11}$ Inferring from a previous study ${ }^{14}$ on ER fluids, the difference in the predicted critical angle may result from its dependency on the particle concentration and the susceptibility, and also from 2D flow assumption. Interestingly, an idealized affine motion of the chain, as reported by Klingenberg and Zukoski, ${ }^{11}$ leading to tilting, breaking, and reforming in a confined shear flow, is observed numerically at the Mason number of $\mathrm{Mn}=0.016$ (Fig. 12(c)). In this regime, the higher the Mason number, the larger the tilting angle of the detached chains to the applied field direction, due to stronger magnetic interactions (see Figs. 12(b) and 12(c) for comparison).

Now, the number of chains is increased to four (see Fig. 13(a)). The area fraction of the particles is equal to $32.4 \%$. In the beginning, the four chains are aligned vertically with both ends in contact with the channel walls. Under continuous shearing, as depicted in Fig. 13, the final conformation of the chains is significantly influenced by the Mason number.

At $\mathrm{Mn}=0.0016$, where the magnetic force is dominant over the viscous force, complex chains with branches are observed rather than linear chains, which is thought to be caused by the strong magnetic interactions among the closely spaced particles, due to the increased particle fraction. The

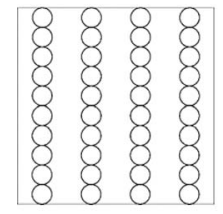

(a)

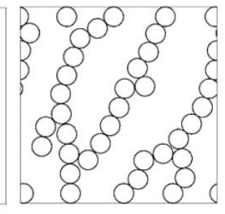

(b)

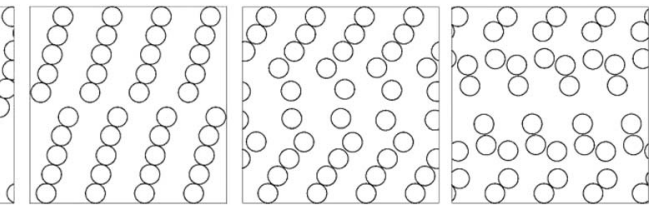

(c) (d) (e)

FIG. 13. Snapshots of typical chain conformations at the four Mason numbers. The number of particles is 40, corresponding to the area fraction of $31.4 \%$. (a) Equally spaced four chains in the beginning, with each chain consisting of 10 particles, and typical chain conformations at (b) $\mathrm{Mn}=0.0016$, (c) $\mathrm{Mn}=0.016$, (d) $\mathrm{Mn}=0.16$, and (c) Mn $=1.6$. (enhanced online) [URL: http://dx.doi.org/10.1063/1.4704822.1] [URL: http://dx.doi.org/10.1063/1.4704822.2]. 
chains are first detached in the middle and each of upper broken chains reconnects with the nearest lower chain approaching to each other. The regular process of breakup and reformation repeats only several steps. Then, the chains are no longer broken in the middle, but broken upper or lower part of the chains. Since then, as depicted in Fig. 13(b), strong magnetic interactions among the detached chains with varying length lead to the formation of complex chains with branches, changing continuously their structures with time (see video 1 in Fig. 13(b)). Since a higher stress is required to break the

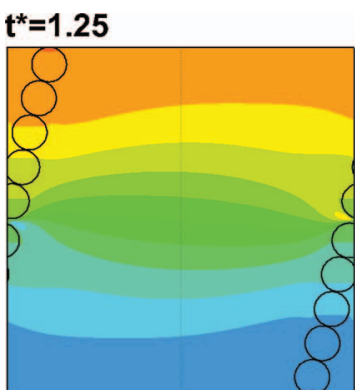

$\mathbf{t}^{*}=\mathbf{2 . 0}$

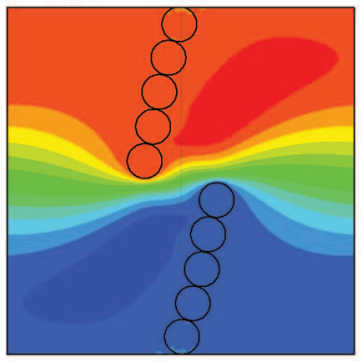

$t^{*}=0.5$

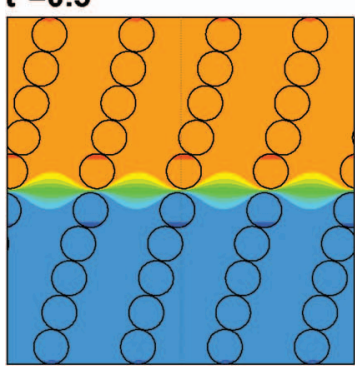

$t^{*}=0.65$

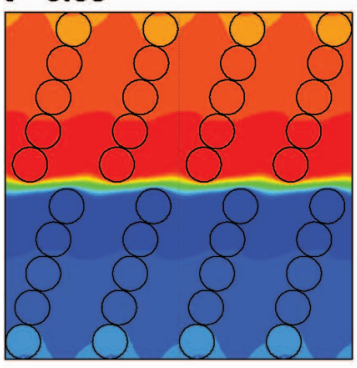

$t^{*}=1.5$

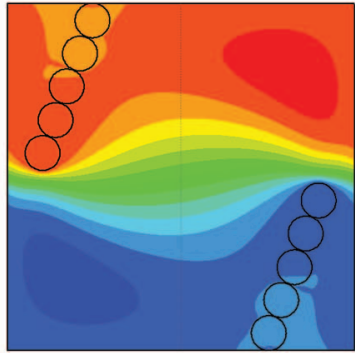

$t^{*}=\mathbf{2 . 2 5}$

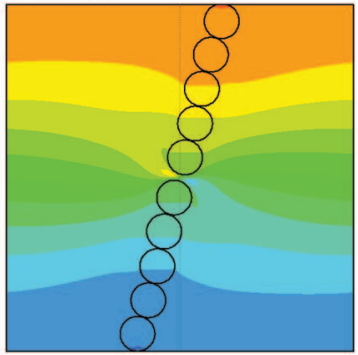

(a)
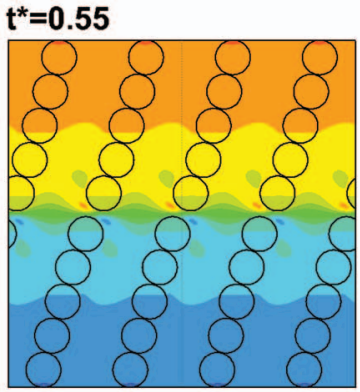

$t^{*}=0.7$

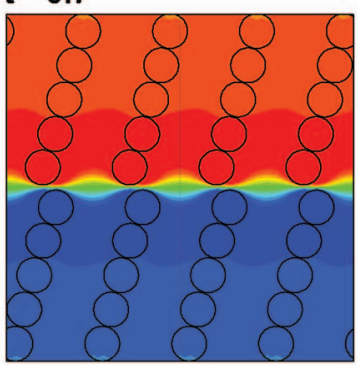

(b)

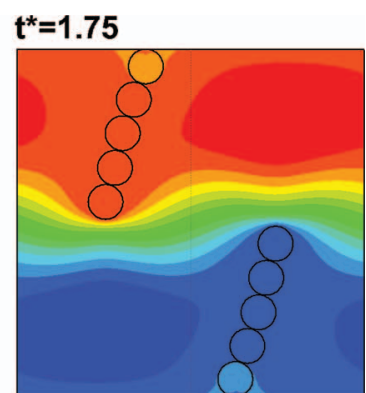

$t^{*}=2.5$

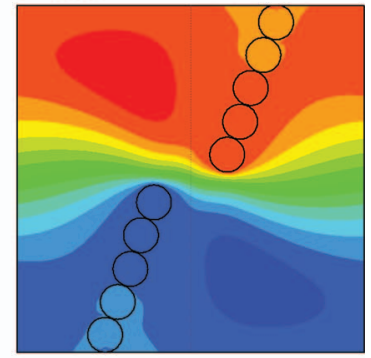

$t^{*}=0.6$

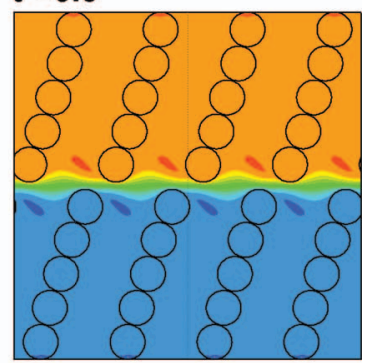

$t^{*}=0.75$

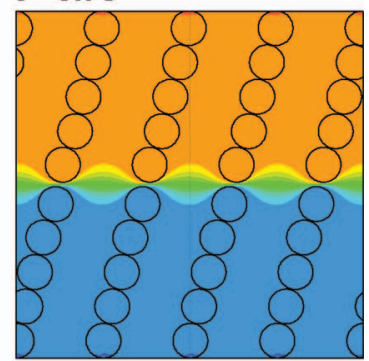

FIG. 14. Contour plots showing the $x$-component of the velocity vector between a rearrangement of the chain conformation for the two cases, (a) single-chain problem and (b) four-chain problem, at $\mathrm{Mn}=0.016$. Contours represent the normalized velocity component, defined by $\tilde{u}=u / U$, where $U$ is the magnitude of the drag velocity imposed on the upper and lower boundaries. Here, $t^{*}$ is the non-dimensional time, defined by $t^{*}=\dot{\gamma} t$. 
chains, the complex chains with branches undergoing continuous rapid breakup and reformation in this small Mason number regime lead to a higher increase in the effective viscosity. Again, at $\mathrm{Mn}=0.016$, the chains are detached in the middle and the detached pair of the chains move in the opposite direction, creating a slip zone at the center of the channel (see video 2 in Fig. 13(c)). These behaviors are the same as those of microstructures of an ER fluid for the dynamic yield stress model reported in the literature. ${ }^{11,14}$ In our simulation, the critical angle $\theta_{c}$ at breakup is approximately $15^{\circ}$, which corresponds to the critical strain $\gamma_{c}=0.26$. The critical strain is smaller than the experimentally observed one varied from 0.4 to $0.6,{ }^{11}$ but more closer to the experimental values than other numerical result ${ }^{13}$ predicting a critical strain of unity. The direct numerical simulation successfully reproduced the particle chaining, tilting, breaking, and reformation at this specific regime of the Mason number, during the shearing of chains of paramagnetic particles in a uniform magnetic field. As the Mason number increases further, shorter chains or single particles fill the channel. At the highest Mason number (chosen in this study), $\mathrm{Mn}=1.6$, a depletion zone with lower concentration of particles is observed in the middle of the channel.

The fluid flow influenced by the evolution of chain conformation is an interesting topic to investigate, especially in the case showing affine motions of chains in the confined shear flow. Therefore, we choose flows at $\mathrm{Mn}=0.016$ to see the effect of chain motions on the fluid flow. Figure 14 depicts the evolution of the $x$-component of the velocity vector during a rearrangement of the chains for the two cases, single- and four-chain problems, with their initial configurations shown in Figs. 12(a) and 13(a), respectively. At the moment of joining, the pair of chains approaching to each other attract with a speed faster than the drag velocity. After breakup, the detached chains swing forward, again with a speed faster than the drag velocity. Due to the swinging motions of the chains, adverse velocity gradient on the wall is observed, especially in the case with higher particle fraction (see Fig. 14(b)). In addition, as reported in the literature, ${ }^{11}$ one can clearly observe a slip zone with locally very high velocity gradient at the center of the channel, when the pairs of chains move to each other. Depicted in Fig. 15 is the $x$-component velocity across the channel at $x=0$ for the chain configurations shown in Fig. 14(b). The extremely steep velocity gradient near the center of the channel, caused by constriction of the shear zone, is the reason of higher effective viscosity in this regime of the Mason number.

In summary, it is found that the magnetoviscous effect in this shear flow is caused by chain conformations highly influenced by the particle fraction and, more importantly, by the interplay between the viscous and magnetic forces characterized by the Mason number. In the latter case with higher particle fraction, three models for electroviscous effect (in this study, magnetoviscous effect)

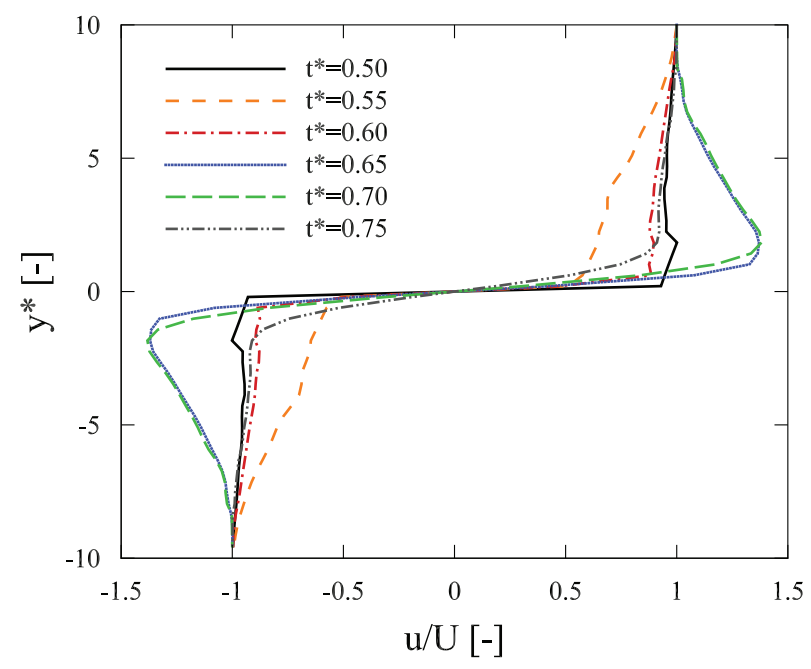

FIG. 15. Plot of the $x$-component velocity across the channel at $x=0$ for the chain configurations shown in Fig. 14(b). In this plot, the abscissa is the normalized velocity, $\tilde{u}=u / U$, and the ordinate is the non-dimensional $y$-coordinate, $y^{*}=y / a$. 
are reproduced by the direct simulation. A specific chain conformation is related to the corresponding range of the Mason number.

\section{CONCLUSION}

We numerically investigated the dynamics of magnetic chains and fluid flow in the presence of both continuous shearing and a uniform magnetic field, with an emphasis on the effect of the Mason number (Mn), the magnetic susceptibility of particles, and particle fraction. A simple shear flow with suspended magnetic chains, under the magnetic field perpendicular to the flow in a two-dimensional periodic channel, was chosen as a model problem.

First, we solved a two-particle problem and found that the rotational dynamics of the chain is governed by the Mason number (the ratio of the viscous force to the magnetic force) for a fixed susceptibility. There exists a critical Mason number, $\mathrm{Mn}_{\mathrm{c}} \sim 0.3$ (based on the definition used), separating the rotational behavior of the chain. Below the critical Mason number, there is an equilibrium between the magnetic torque and viscous torque, leading to finite rotation of the chain. In this regime, the angular velocity of each particle approaches zero as well. In the higher Manson number regime, where $\mathrm{Mn}>\mathrm{Mn}_{\mathrm{c}}$, however, the rotational motion of the particles is time periodic and the fluid flow as well. Regardless of the Mason number, the two particles move like a single rigid chain; thus, the angular velocity of the chain is the same as that of each particle.

Streamlines obtained from the perturbed velocity due to the presence of two particles may be either hyperbolic or elliptic in nature, depending on the Mason number. At lower Mason numbers, $\mathrm{Mn}<\mathrm{Mn}_{\mathrm{c}}$, the perturbed flow is elliptic. Above the critical value, however, hyperbolic perturbed flow is observed around the chain of two particles. If suspended chains are unconstrained by the wall, below the threshold value of Mn, the non-dimensional wall shear stress is almost constant. While, above the threshold value, a thinning behavior in the wall stress is observed due to higher tilting angle of the chains, resulting in lower flow resistance.

As for chain rupture in the shear flow, three regimes of the Mason number were found, showing different chain conformations. At smaller Mason numbers $(\mathrm{Mn}<0.01)$, where the magnetic force is dominant, complex chains with branches are observed rather than linear chains as the particle fraction increases. At intermediate regime (around $\mathrm{Mn}=0.01$ ), the initially intact chains are detached in the middle and the detached pair of chains move in the opposite direction, creating a narrow slip zone with a very steep velocity gradient. As the Mason number increases further, shorter chains or single particles fill the channel. A depletion zone with lower particle concentration is observed at even higher Mason numbers.

\section{ACKNOWLEDGMENTS}

This research was supported by 2008 Korea Aerospace University faculty research (Grant No. 2008-01-004) and Basic Science Research Program through the National Research Foundation of Korea (NRF) funded by the Ministry of Education, Science and Technology (Grant No. 20100004802).

${ }^{1}$ D. J. Klingenberg, "Magnetorheology: Applications and challenges," AIChE J. 47, 246-249 (2001).

${ }^{2}$ F. D. Goncalves, J.-H. Koo, and M. Ahmadia, "A review of the state of the art in magnetorheological fluid technologies Part I: MR fluid and MR fluid models," Shock Vibration Digest 38, 203-219 (2006).

${ }^{3}$ H. Suzuki, C.-M. Ho, and N. Kasagi, "A chaotic mixer for magnetic bead-based micro cell sorter," J. Microelectromech. Syst. 13, 779-790 (2004).

${ }^{4}$ M. A. M. Gijs, "Magnetic bead handling on-chip: New opportunities for analytical applications," Microfluid Nanofluid 1, 22-40 (2004).

${ }^{5}$ A. Rida and M. A. M. Gijs, "Manipulation of self-assembled structures of magnetic beads for microfluidic mixing and assaying," Anal. Chem. 76, 6239-6246 (2004).

${ }^{6}$ N. Pamme, "Magnetism and microfluidics," Lab. Chip. 6, 24-38 (2006).

${ }^{7}$ R. Calhoun, A. Yadav, P. Phelan, A. Vuppu, A. Garciab, and M. Hayes, "Paramagnetic particles and mixing in micro-scale flows," Lab. Chip. 6, 247-257 (2006).

${ }^{8}$ T. G. Kang, M. A. Hulsen, P. D. Anderson, J. M. J. den Toonder, and H. E. H. Meijer, "Chaotic mixing induced by a magnetic chain in a rotating magnetic field," Phys. Rev. E 76, 066303 (2007). 
${ }^{9}$ I. Petousis, E. Homburg, R. Derks, and A. Dietzel, "Transient behaviour of magnetic micro-bead chains rotating in a fluid by external fields," Lab Chip 7, 1746-1751 (2007).

${ }^{10}$ A. Ranzoni, X. J. A. Janssen, M. Ovsyanko, L. J. van IJzendoorn, and M. W. J. Prins, "Magnetically controlled rotation and torque of uniaxial microactuators for lab-on-a-chip applications," Lab Chip 10, 179-188 (2010).

${ }^{11}$ D. J. Klingenberg and C. F. Zukoski, "Studies on the steady-shear behavior of electrorheological suspensions," Langmuir 6, 15-24 (1990).

${ }^{12}$ T. C. Halsey, J. E. Martin, and D. Adolf, "Rheology of electrorheological fluids," Phys. Rev. Lett. 68, 1519-1522 (1992).

${ }^{13}$ R. T. Bonnecaze and J. F. Brady, "Dynamic simulation of an electrorheological fluid," J. Chem. Phys. 96, 2183-2202 (1992).

${ }^{14}$ R. T. Bonnecaze and J. F. Brady, "Yield stresses in electrorheological fluids," J. Rheol. 36, 73-115 (1992).

${ }^{15}$ J. E. Martin, J. Odinek, T. C. Halsey, and R. Kamien, "Structure and dynamics of electrorheological fluids," Phys. Rev. E 57, 756-775 (1998).

${ }^{16}$ J. de Vicente, M. T. López-López, J. D. G. Durán, and F. González-Caballero, "Shear flow behavior of confined magnetorheological fluids at low magnetic field strengths," Rheol. Acta 44, 94-103 (2004).

${ }^{17}$ S. Melle and J. E. Martin, "Chain model of a magnetorheological suspension in a rotating field," J. Chem. Phys. 118, 9875-9881 (2003).

${ }^{18}$ S. Melle, O. G. Calderon, M. A. Rubio, and G. G. Fuller, "Microstructure evolution in magnetorheological suspensions governed by Mason number," Phys. Rev. E 68, 041503 (2003).

${ }^{19}$ T. G. Kang, M. A. Hulsen, J. M. J. den Toonder, P. D. Anderson, and H. E. H. Meijer, "A direct simulation method for flows with suspended paramagnetic particles," J. Comput. Phys. 227, 4441-4458 (2008).

${ }^{20}$ S. Kang and Y. K. Suh, "An immersed-boundary finite-volume method for direct simulation of flows with suspended paramagnetic particles,” Int. J. Numer. Meth. Fluids 67, 58-73 (2011).

${ }^{21}$ J. A. Stratton, Electromagnetic Theory (McGraw-Hill Book Company Inc., New York, 1941).

${ }^{22} \mathrm{C}$. Rinaldi and H. Brenner, "Body versus surface forces in continuum mechanics: Is the maxwell stress tensor a physically objective cauchy stress?" Phys. Rev. E 65, 036615 (2002).

${ }^{23}$ R. Glowinski, T. W. Pan, T. I. Hesla, and D. D. Joseph, “A distributed lagrange multiplier/fictitious domain method for particulate flows,” Int. J. Multiphase Flow 25, 755-794 (1999).

${ }^{24}$ T. B. Jones, Electromechanics of Particles (Cambridge University Press, New York, 1995).

${ }^{25}$ J. Jin, The Finite Element Method in Electromagnetics (Wiley, New York, 2002).

${ }^{26}$ W. R. Hwang, M. A. Hulsen, and H. E.H. Meijer, "Direct simulation of particle suspensions in sliding bi-periodic frames," J. Comput. Phys. 194, 742-772 (2004).

${ }^{27}$ I. S. Duff, "MA57-A code for the solution of sparse symmetric definite and indefinite systems," ACM Trans. Math. Softw. 30, 118-144 (2004).

${ }^{28}$ X. Wang, X.-B. Wang, and P. R. C. Gascoyne, "General expressions for dielectrophoretic force and electrorotational torque derived using the Maxwell stress tensor method," J. Electrostat. 39, 277-295 (1997).

${ }^{29}$ G. B. Jeffery, "The motion of ellipsoidal particles immersed in a viscous fluid," Proc. R. Soc. A 102, 161-179 (1922).

${ }^{30}$ W. R. Hwang, P. D. Anderson, and M. A. Hulsen, "Chaotic advection in a cavity flow with rigid particles," Phys. Fluids 17, 043602 (2005). 